\title{
Chromatic Dispersion-Aware Carrier-Phase Estimation for Digital Subcarrier Multiplexing Systems
}

\author{
Manuel S. Neves, Paulo P. Monteiro and Fernando P. Guiomar
}

Instituto de Telecomunicações and University of Aveiro, Campus Universitário de Santiago, 3810-193, Portugal.

\begin{abstract}
We propose a novel Dual-Reference Subcarrier (DRS) algorithm for improved carrier-phase estimation in long-haul Multi-Subcarrier (MSC) systems. For a 64 Gbaud 64QAM MSC signal, the DRS algorithm is shown to enable penalty-free operation with symbol-rates per subcarrier as low as 2 Gbaud.
\end{abstract}

\section{Introduction}

Digital subcarrier multiplexing systems have been gaining both academic and commercial interest lately due to their associated advantages ${ }^{[1]-[6]}$, namely in terms of inherent DSP parallelism, enhanced bit-rate granularity ${ }^{[2],[3]}$ and higher tolerance against Chromatic Dispersion (CD $)^{[4]}$, Nonlinear Interference Noise $(\mathrm{NLIN})^{[5],[6]}$ and Equalization-Enhanced Phase Noise (EEPN) ${ }^{[1]}$. However, they also still present an important disadvantage from the Carrier Phase Estimation (CPE) point of view, due to the increased symbol periods ${ }^{[1]}$. In order to try and circumvent this limitation, several joint-subcarrier CPE schemes have been proposed ${ }^{[7]-[9]}$, but these solutions are not specifically aimed at maintaining the increased tolerance to CD that is brought by MSC systems. In fact, when affected by $C D$, these joint-subcarrier CPE algorithms can even perform worse than plain per-subcarrier approaches. Having this in mind, in this paper we devise a novel CD-aware joint-subcarrier algorithm, which is shown to allow for penalty-free operation with symbol-rates in the Gbaud range.

\section{Dual-Reference Subcarrier CPE Algorithm}

Figure 1 shows a block diagram depicting the interplay between Laser Phase Noise (LPN) and $C D$ in a long-haul coherent optical system, assuming a linear fiber channel. In dispersion-less scenarios, the LPNs inserted by the transmitter, $\phi_{\mathrm{TX}}$, and Local Oscillator (LO), $\phi_{\mathrm{LO}}$, can effectively be combined as a single Wiener phase noise. However, the effect of CD during propagation makes the receiver-side treatment of those two independent LPN sources more complex. Each frequency component of the transmitted signal travels at a different group velocity over the fiber, arriving at different time instants to the receiver. This causes each frequency to be affected by a different overall LPN, resulting from the sum of two Wiener processes shifted in time.

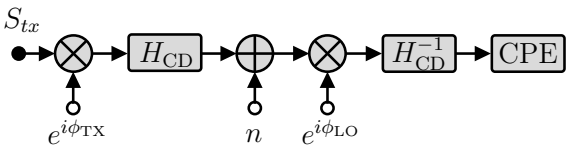

Fig. 1: Simplified block diagram of the relevant effects for joint-subcarrier CPE in the presence of CD $\left(H_{\mathrm{CD}}\right)$.

Let us now consider a signal composed of $N_{\mathrm{SC}}$ narrow-band subcarriers (such that we can ignore intra-subcarrier CD effects) and ideal zero-forcing Chromatic Dispersion Equalization (CDE). In this scenario, each subcarrier is affected by an overall LPN, $\phi_{\mathrm{PN} j}$, that can accurately be described by a linear combination of $\phi_{\mathrm{TX}}$ and $\phi_{\mathrm{LO}}$,

$$
\phi_{\mathrm{PN} j}(t)=\phi_{\mathrm{TX}}(t)+\phi_{\mathrm{LO}}\left(t-\left(\frac{N_{\mathrm{SC}}+1}{2}-j\right) T_{\mathrm{CD}}\right),
$$

where $j \in 1,2, \ldots, N_{\mathrm{SC}}$ is the subcarrier index and $T_{\mathrm{CD}}=L \beta_{2} \Delta \omega$ represents the walk-off effect incurred by CD between two adjacent subcarriers, where $\beta_{2}$ is the group velocity dispersion parameter, $L$ is the fiber propagation length and $\Delta \omega$ is the angular frequency spacing between subcarriers. It then becomes apparent from expression (1) that the realization of an effective joint-subcarrier CPE in this scenario must take into consideration the distributed (TX and LO) origin of LPN. Whereas current joint-subcarrier CPE approaches tend to rely on the direct estimation of the overall LPN, in this work we propose to estimate $\phi_{\mathrm{TX}}$ and $\phi_{\mathrm{LO}}$ separately, and only then properly combine them to correct the impact of LPN in each subcarrier.

Let us now start by considering two reference subcarriers, $\mathrm{SC}_{m}$ and $\mathrm{SC}_{n}$, whose individual LPN estimates are represented by $\hat{\phi}_{\mathrm{PN} m}$ and $\hat{\phi}_{\mathrm{PN} n}$, respectively. By applying expression (1), it is interesting to note that the subtraction of these two LPN estimates allows to cancel out the contribution of $\phi_{\mathrm{TX}}$,

$$
\begin{aligned}
& \hat{\phi}_{\mathrm{PN} m}(k)-\hat{\phi}_{\mathrm{PN} n}(k)= \\
& \phi_{\mathrm{LO}}\left(k T_{s}-\left(\frac{N_{\mathrm{SC}}+1}{2}-m\right) T_{\mathrm{CD}}\right)- \\
& \phi_{\mathrm{LO}}\left(k T_{s}-\left(\frac{N_{\mathrm{SC}}+1}{2}-n\right) T_{\mathrm{CD}}\right)=\Delta \phi_{\mathrm{LO}}(k),
\end{aligned}
$$




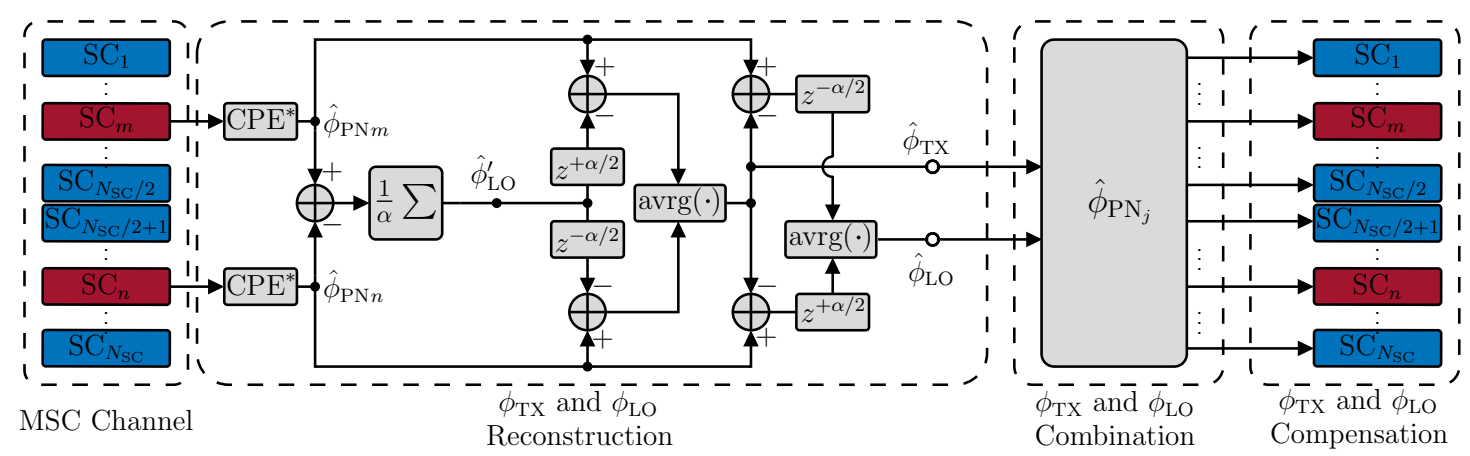

Fig. 2: Block diagram of the DRS algorithm. The "CPE*" block represents a LPN estimate extraction from a subcarrier, resorting to a pilot-based approach.

where $T_{s}$ is the symbol period and $\Delta \phi_{\mathrm{LO}}$ represents the temporal evolution of $\phi_{\mathrm{LO}}$ in the next $\left|(m-n) T_{\mathrm{CD}}\right|$ seconds. By assuming a linear evolution of $\phi_{\mathrm{LO}}$ within the $\left|(m-n) T_{\mathrm{CD}}\right|$ period, it follows that,

$$
\phi_{\mathrm{LO}}(k)-\phi_{\mathrm{LO}}(k-1)=\frac{1}{\alpha} \Delta \phi_{\mathrm{LO}}(k),
$$

with $\alpha$ being an adjustment factor given by,

$$
\alpha=\frac{(m-n) T_{\mathrm{CD}}}{T_{s}} .
$$

An estimate of the instantaneous LPN impinging on the LO, $\hat{\phi}_{\mathrm{LO}}^{\prime}$, can now be obtained by integrating expression (3) as,

$$
\hat{\phi}_{\mathrm{LO}}^{\prime}(k)=\frac{1}{\alpha} \sum_{k^{\prime}=1}^{k} \Delta \phi_{\mathrm{LO}}\left(k^{\prime}\right)+\phi_{\mathrm{LO}}(1),
$$

where $\phi_{\mathrm{LO}}(1)$ is a constant phase shift that can be safely ignored. This initial estimation of the isolated effect of the LO phase noise, $\hat{\phi}_{\mathrm{LO}}^{\prime}$, is the central point for the dual-reference subcarrier (DRS) algorithm proposed in this work, whose implementation diagram is depicted in Fig. 2. As shown in the " $\phi_{\mathrm{TX}}$ and $\phi_{\mathrm{LO}}$ Reconstruction" block of Fig. 2, an estimate of the transmitter LPN, $\hat{\phi}_{\mathrm{TX}}$, can now be obtained by removing a properly aligned version of $\hat{\phi}_{\mathrm{LO}}^{\prime}$ from the overall LPN estimates of the two reference subcarriers. Finally, a second refined estimate of $\phi_{\mathrm{LO}}, \hat{\phi}_{\mathrm{LO}}$, can be obtained by subtracting $\hat{\phi}_{\mathrm{TX}}$ from both $\hat{\phi}_{\mathrm{PN} m}$ and $\hat{\phi}_{\mathrm{PN} n}$. After obtaining the $\hat{\phi}_{\mathrm{TX}}$ and $\hat{\phi}_{\mathrm{LO}}$ estimates, the LPN affecting each subcarrier can be straightforwardly reconstructed through expression (1), and then LPN compensation can be applied on a per-subcarrier basis.

\section{Numerical Performance Assessment}

Considering a 64QAM Root-Raised-Cosine (RRC) (5\% roll-off factor) modulated at 64 Gbaud, we will now numerically assess the performance of the DRS algorithm under different conditions of combined laser linewidth and accumulated dispersion. Assuming a Forward Error Correction (FEC) rate of $5 / 6$ ( $20 \%$ overhead), the DRS performance will be assessed in terms of required Signal to Noise Ratio (SNR) to achieve a realistic Normalized Generalized Mutual Information (NGMI) of 0.9. As a performance benchmark, the DRS algorithm will be compared against a pilot-based implementation on a per-subcarrier basis $(P)$. For the per-subcarrier pilot-based implementation, the pilot-rate, $R_{\mathrm{P}}$, is $31 / 32$ on all subcarriers, whereas for the DRS algorithm all pilots are concentrated on the two reference subcarriers, while maintaining the same overall $R_{\mathrm{P}}=31 / 32$, thus yielding the same net bit-rate. In all cases, the number of CPE taps for noise averaging has been optimized.

The DRS algorithm requires, as the name implies, the election of two reference subcarriers. In order to minimize the distance between reference and non-reference subcarriers, we start by considering $m=\frac{N_{\mathrm{SC}}}{4}+1$ and $n=\frac{3 N_{\mathrm{SC}}}{4}-1$ as the designated reference subcarriers.

Figure 3a shows a sweep of laser linewidth for a fixed transmission length of $2000 \mathrm{~km}$. We present the results of subcarrier-independent pilot-based CPE and DRS algorithms for 4, 8, and 32 SCs. In all cases, the DRS algorithm is shown to clearly outperform the best subcarrierindependent pilot-based approach, while enabling an ever-increasing performance with higher SC count, thus overcoming the well-known degradation of MSC performance with lower SC symbol rates ${ }^{[1]}$. It is worth noting that the gain provided by the DRS algorithm is further evidenced for large combined linewidths. Note that, while the subcarrier-independent pilot-based CPE requires a maximum of 4 SCs (16 Gbaud per SC) to minimize the penalty with combined laser linewidths above $500 \mathrm{kHz}$, the DRS algorithm allows for 

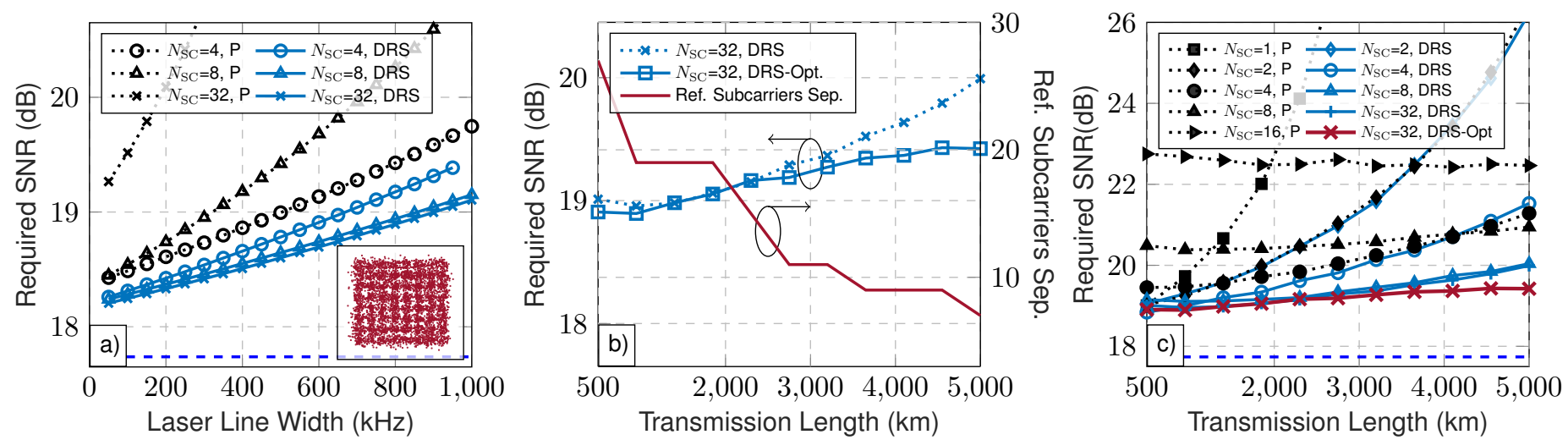

Fig. 3: Performance of the DRS CPE, using a 64QAM RRC (5\% roll-off factor) modulated at 64 Gbaud. a) dependence on laser linewidth for $L=2000 \mathrm{~km}$; b) impact of accumulated CD on the optimum separation between reference subcarriers, for $1 \mathrm{MHz}$ combined laser linewidth; c) dependence on transmission length for a laser linewidth of $1 \mathrm{MHz}$.

penalty-free operation with $N_{\mathrm{SC}}=32$, corresponding to 2 Gbaud per SC.

Let us now focus on the specific case of $N_{\mathrm{SC}}=32$ with $1 \mathrm{MHz}$ combined laser linewidth. In Fig. 3b, we can see how, for the evaluated scenario, the performance of the DRS algorithm still presents some dependence on the transmission length (SSMF with dispersion parameter of $20.4 \mathrm{ps} / \mathrm{nm} / \mathrm{km}$ ). After revising the details of the algorithm, we can note how the adjustment factor, $\alpha$, described on expression (4) imposes a penalty in the estimation of $\phi_{\mathrm{LO}}$. In fact, $\alpha$ corresponds to the number of symbols over which an estimation of a value of $\phi_{\mathrm{LO}}(t)$ is spread, and therefore it produces a filtering effect. To change the value of $\alpha$, we need to change the separation between reference SCs. Note that by setting $m=\frac{N_{\mathrm{SC}}}{4}+1$ and $n=\frac{3 N_{\mathrm{SC}}}{4}-1$, the default separation between reference $\mathrm{SCs}$ is equal to $N_{\mathrm{SC}} / 2-2$. If we reduce this separation by bringing reference SCs closer to the DC, we will be reducing the spreading effect, further increasing the performance for longer transmission lengths. In Fig. $3 \mathrm{~b}$ the solid blue line shows the results of optimizing the distance between reference SCs (DRS-Opt.), and the resultant optimized reference SC separation is also shown by the red solid line. At a transmission length of $5000 \mathrm{~km}$, we obtain an optimum separation between reference SCs of $m-n=6$, yielding a gain of around $0.6 \mathrm{~dB}$ over the initially designated reference SCs, and there is a visible reduction on the slope of the overall DRS performance curve. As a rule of thumb, we might assert that the evenly spaced $m=\frac{N_{\mathrm{SC}}}{4}+1$ and $n=\frac{3 N_{\mathrm{SC}}}{4}-1$ reference SCs can be safely utilized for long-haul distances up to $2000-3000 \mathrm{~km}$, whereas the subcarrier spacing should be further reduced for ultra-long-haul distances.
Finally, let us perform a broader set of tests in a distance sweep, for a fixed combined laser linewidth of $1 \mathrm{MHz}$. Figure 3c shows these results. Starting our analysis with the results of the subcarrier-independent pilot-based CPE, we can see how there seems to be a clear disadvantage: the number of subcarriers of the MSC system has to be changed as a function of the intended transmission distance for optimum performance. Now, analyzing the results of the DRS algorithm, we observe that it yields the best performance with $N_{\mathrm{SC}}=32$ for the entire range of transmission lengths. Besides simplifying the design of the MSC signal, this also makes it possible to benefit from the inherent practical advantages of using a larger number of digital subcarriers.

\section{Conclusions}

We have proposed a novel CD-aware dualreference subcarrier $\mathrm{CPE}$, which was shown to enable penalty-free carrier-phase estimation for long-haul optical systems composed of low baudrate subcarriers (2 Gbaud per SC), thereby unlocking the full advantages of digital subcarrier multiplexing, namely in terms of DSP parallelism, bit-rate granularity and enhanced nonlinearity tolerance. Future work comprises the extension of the DRS technique to the mitigation of nonlinear phase noise, and the realization of independent digital monitoring of TX and LO laser linewidths.

\section{Acknowledgements}

This work was partially supported by FEDER, through the CENTRO 2020 programme, project ORCIP (CENTRO01-0145-FEDER-022141), and by FCT/MCTES through project FreeComm-B5G (UIDB/EEA/50008/2020). Fernando P. Guiomar acknowledges a fellowship from "la Caixa" Foundation (ID 100010434). The fellowship code is LCF/BQ/PR20/11770015. 


\section{References}

[1] H. Sun, M. Torbatian, M. Karimi, R. Maher, S. Thomson, M. Tehrani, Y. Gao, A. Kumpera, G. Soliman, A. Kakkar, M. Osman, Z. A. El-Sahn, C. Doggart, W. Hou, S. Sutarwala, Y. Wu, M. R. Chitgarha, V. Lal, H. Tsai, S. Corzine, J. Zhang, J. Osenbach, S. Buggaveeti, Z. Morbi, M. Iglesias Olmedo, I. Leung, X. Xu, P. Samra, V. Dominic, S. Sanders, M. Ziari, A. Napoli, B. Spinnler, K. Wu, and P. Kandappan, "800G DSP ASIC design using probabilistic shaping and digital sub-carrier multiplexing", Journal of Lightwave Technology, vol. 38, no. 17, pp. 4744-4756, 2020. DOI: 10.1109/JLT. 2020. 2996188.

[2] F. P. Guiomar, L. Bertignono, A. Nespola, and A. Carena, "Frequency-domain hybrid modulation formats for high bit-rate flexibility and nonlinear robustness", Journal of Lightwave Technology, vol. 36, no. 20, pp. 4856-4870, 2018. DOI: 10.1109/JLT. 2018. 2866625.

[3] M. Cantono, F. P. Guiomar, A. Carena, and V. Curri, "Networking benefit of multi-subcarrier transceivers", in Proc. Optical Fiber Communications Conference and Exposition (OFC), 2018, Tu3E.1. [Online]. Available: https : // ieeexplore. ieee.org/document/8385962.

[4] M. Malekiha, I. Tselniker, and D. V. Plant, "Chromatic dispersion mitigation in long-haul fiber-optic communication networks by sub-band partitioning", Opt. Express, vol. 23, no. 25, pp. 32654-32 663, 2015. DOI: 10.1364/ OE. 23.032654.

[5] P. Poggiolini, A. Nespola, Y. Jiang, G. Bosco, A. Carena, L. Bertignono, S. M. Bilal, S. Abrate, and F. Forghieri, "Analytical and experimental results on system maximum reach increase through symbol rate optimization", Journal of Lightwave Technology, vol. 34, no. 8, pp. 1872-1885, 2016. DOI: 10 . 1109 / JLT . 2016 . 2516398.

[6] M. Qiu, Q. Zhuge, M. Chagnon, Y. Gao, X. Xu, M. MorsyOsman, and D. V. Plant, "Digital subcarrier multiplexing for fiber nonlinearity mitigation in coherent optical communication systems", Opt. Express, vol. 22, no. 15, pp. 18770-18777, Jul. 2014. DOI: 10 . 1364 / OE . 22 . 018770.

[7] S. M. Bilal, C. Fludger, and G. Bosco, "Carrier phase estimation in multi-subcarrier coherent optical systems", IEEE Photonics Technology Letters, vol. 28, no. 19, pp. 2090-2093, 2016. DOI: 10 . 1109 / LPT . 2016 . 2585500.

[8] M. P. Yankov, L. Barletta, and D. Zibar, "Low-complexity joint sub-carrier phase noise compensation for digital multi-carrier systems", in Proc. European Conference on Optical Communication (ECOC), 2017. DOI: 10.1109/ ECOC. 2017.8345922.

[9] D. Martinez, Y. Mori, H. Hasegawa, and K.-i. Sato, "Novel subcarrier multiplexing and subcarrier-synchronous phase estimation tolerant to laser phase noise", in Signal Processing in Photonic Communications (SPPCom), 2017, SpW1F.5. DOI: 10.1364/SPPCOM. 2017.SpW1F.5. 